\title{
A Simple Technique for Reduction of Nearly Extruded Talar Body in Hawkin's Type III Fracture Neck Talus
}

\author{
Amr Farouk Abdel Rahman*, Mohamed Mokhtar Abd-Ella and Mohammed Zayan Ibrahim \\ Department of Orthopedic Surgery, Ain Shams University, Egypt
}

Submission: August 31, 2016; Published: September 16, 2016

*Corresponding author: Amr Farouk Abdel Rahman, Lecturer of Orthopedic surgery, Ain Shams University, Demerdash Hospital, 56 Ramsis street, Abbaseyya square, Egypt, Tel: +20 1007090067; + 202 26844195; Email: amrbekfarouk@hotmail.com

\begin{abstract}
Background: Avascular necrosis of talar body and osteoarthristis of ankle and subtalar joints are very common complications in fracture neck talus. Early and prompt reduction of the extruded talar body and its adequate fixation is mandatory to save the delicate blood supply and the articular cartilage. Obstacles against reduction include tight mortis, entrapped tendons requiring vigorous manipulation that leads to soft tissue insult, articular cartilage scuffing, and propagation of undisplaced cracks adding more comminuted fragments.

Aim: To propose a distraction technique using a simple ring fixator to achieve anatomical easy reduction of extruded talar body without vigorous manipulation.

Methods: The distraction technique was conducted for 6 patients (four males and two females), mean age 28 years. All of them had fracture neck talus Hawkin's type 3 with nearly extruded talar body. Simple two level ring fixator was applied for all patients, reduction was achieved and assessed by image intensifier then fixation was done by 2 cannulated $4 \mathrm{~mm}$ screws. Post operative $\mathrm{x}$ rays and CT scan were done for all patients. Partial weight bearing started at 4 weeks. Fixator was removed at 12 weeks, follow up period lasted for 12 months, functional scoring using AOFAS scoring was done to assess the patients at last follow up.

Results: Good reduction was achieved in all cases, two cases had superficial wound dehiscence treated by intravenous antibiotics and frequent dressing, two cases were complicated by Avascular necrosis that required no further intervension, one case required bone grafting for delayed union, mean AOFAS scoring system was 79.5 out of 100 at last follow up.

Conclusion: Distraction assisted reduction of extruded talar body is a good proposed technique for treatment of Hawkin's type 3 fractures of neck talus without need for vigorous manipulation.
\end{abstract}

\section{Introduction}

Although cases of talar fractures are not common, comprising only $3 \%$ of foot fractures, it is considered a very challenging type of fractures. Anatomically, talar fractures can be divided into body fractures, neck fractures, head fractures, lateral process fractures and posterior process fractures, of which neck fractures make up 50\% [1]. Hawkin's classification which is mostly used to classify talar neck fractures consists of four types. Type I is non-displaced fracture. Type II is assosciated with subtalar subluxation or dislocation. Type III is assosciated with subtalar and ankle dislocation. Type IV is a type III with associated talonavicular subluxation or dislocation [2]. Avascular necrosis of the talar body is a common complication after talar neck fracture. Its incidence is directly proportional to the Hawkin's grade, with incidence from zero to $15 \%$ in type I and incidence reaching $100 \%$ in type IV.

The incidence in type II and III ranges from 15 to $75 \%[2,3]$ (Tables $1 \& 2$ ). Early and prompt reduction with adequate fixation of talar neck fracture dislocation is necessary in trying to save the delicate blood supply of the talus [4]. Operative reduction of the dislocated talar body is sometimes so difficult due to contracted soft tissues in delayed cases or entrapped tendons as tibialis posterior and flexor hallucis longus so, the aim of this study is to evaluate the role of distraction using Ilizarov frame in facilitating open reduction of dislocated talar body in cases of talar neck fracture dislocation.

Table 1: Hawkin's Classification.

\begin{tabular}{|c|c|}
\hline Type I & Non-displaced talar neck fracture \\
\hline Type II & $\begin{array}{r}\text { Talar neck fracture with mild displacement. Subluxing of } \\
\text { subtalar joint }\end{array}$ \\
\hline Type III & $\begin{array}{r}\text { Talar neck fracture with moderate displacement. } \\
\text { Subluxing of subtalar and ankle joint }\end{array}$ \\
\hline Type IV & $\begin{array}{r}\text { Talar neck fracture with severe displacement. Sublaxing } \\
\text { of subtalar, ankle, and talonavicular joint }\end{array}$ \\
\hline
\end{tabular}


Table 2: Associated injuries.

\begin{tabular}{|c|c|}
\hline 1 patient & Upper limb (humerus) fracture \\
\hline 1 patient & Fracture ribs \\
\hline 1 patient & Polytrauma (ipsilateral femur, lumar spine) \\
\hline 3 patients & Medial malleolar fracture \\
\hline 1 patient & Posterior tibial artery compromise \\
\hline
\end{tabular}

\section{Materials and Methods}
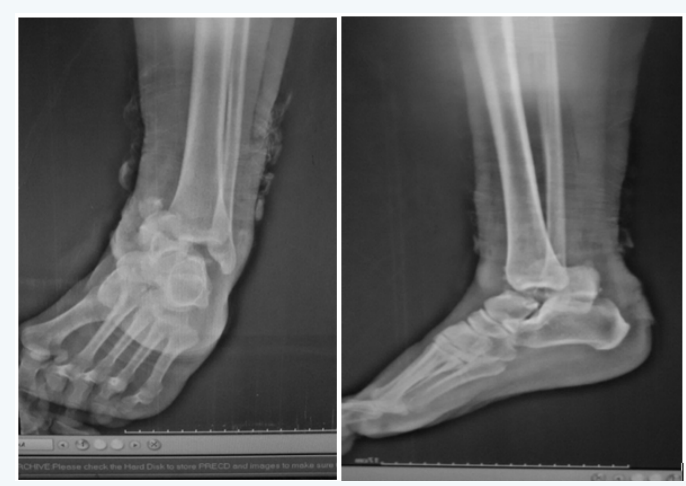

Figure 1: Preoperative $X$ rays of a patient with Hawkin's type 3 talar neck fracture and associated medial malleolar fracture.

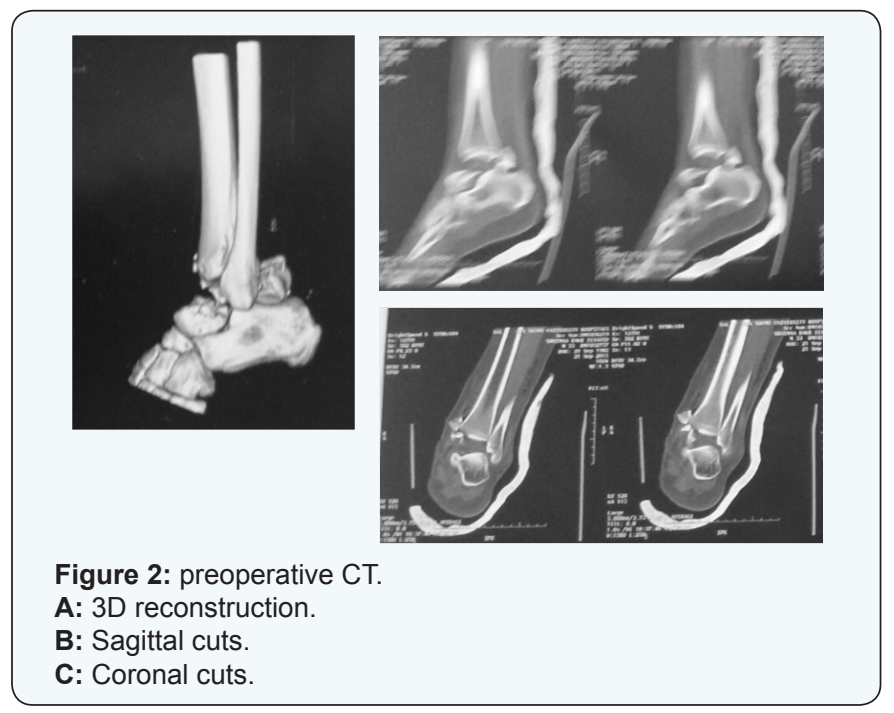

The new technique was conducted between 2014 to 2016 in Ain Shams University Hospitals on six patients, four males and two females. All of them were between $3^{\text {rd }}$ and $5^{\text {th }}$ decade (mean age 28 years). All of them had Hawkin's type III talar neck fracture with nearly extruded talar body (Figures $1 \& 2$ ). The mechanism of injury was road traffic accident in four cases and fall from height in two cases. Two cases were open fractures Gustillo grade 2, and four patients had closed injury. One case had associated upper limb fracture, another case had associated rib fracture and one patient was a polytrauma patient with associated fracture lumbar spine and ipsilateral femur (Table 3 ), three cases had associated medial malleolar fracture [5]. One of the patients had vascular compromise with the posterior tibial artery could not be palpated or heard by Doppler. Average time elapsed between trauma and surgical intervention ranged between 4 hours and 24 hours me elapsed between trauma.

Table 3: Complications.

\begin{tabular}{|l|l|}
\hline 2 patients & Superficial wound dehiscence \\
\hline 1 patient & Delayed union required graft at 6 months \\
\hline 2 patients & Radiological signs of AVN \\
\hline
\end{tabular}

\section{Surgical technique}

Patient put in supine position, above knee tourniquet was applied. Approach used is double anteromedial and anterolateral approach. Anteromedial approach is made between tibialis posterior and tibialis anterior tendons [6] (in both patients with open fractures wounds were on medial aspect of the ankle and were extended slightly proximally and distally following the line of anteromedial approach), in patients with associated medial malleolar fracture, it was not fixed except after fixing the talar fracture to make use of the good exposure created by the malleolar fracture while in patients with intact medial malleolus osteotomy was not needed.

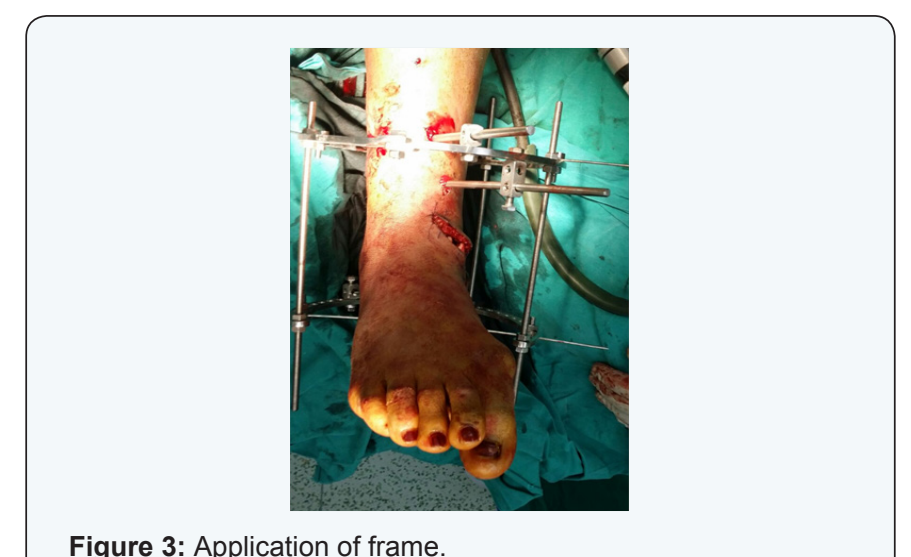

Figure 3: Application of frame.

Anterolateral approach is made anterior to the lateral malleolus extending towards the fourth metatarsal avoiding branches of the superficial peroneal nerve [6], this incision is used to double check the reduction of the talus and in introducing one of both cannulated screws. Applying a ring fixator frame formed of one ring put at fifteen $\mathrm{cm}$ proximal and parallel to the ankle joint line and fixed to the tibia with two wires and two (five $\mathrm{mm}$ ) shanz pins, the distal foot piece is a 5/8 ring that is fixed to the calcaneus by two wires and one (five $\mathrm{mm}$ ) shanz pin (Figure 3 ). Both rings should be parallel to the joint line, wires are tensioned and four connecting rods are put across both rings.

Distraction is now done equally across the four connecting rods, distraction can be increased up to five $\mathrm{cm}$ and its extent 


\section{Orthopedics and Rheumatology Open Access Journal}

is checked using the image intensifier (Figure 4), excessive distraction should be avoided even if possible specially in cases with vascular compromise, the sign for adequate distraction is the clear vision of the articular cartilage of the distal tibia and the posterior calcaneal facet through the anteromedial incision, while the talar body is still extruded from the mortis. Entrapped tendons of the tibialis posterior and flexor hallucis should be cleared from the mortis through the medial incision to pave a safe way for reducing the talar body back to the mortis, this reduction can be now done very gently using one finger without need for any vigorous manipulation .
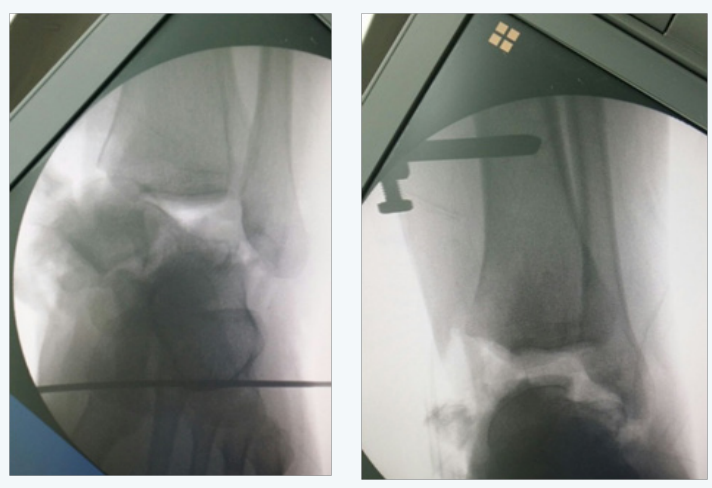

Figure 4: Distraction under image intensifier.

In order to maintain the reduced talar body inside the mortis the distraction has to be reversed across the four rods partially till the distal tibia touches the talar dome.

Reduction is assessed by:

a. Image intensifier in Anteroposterior view, lateral view, and Mortis view (Figure 5).
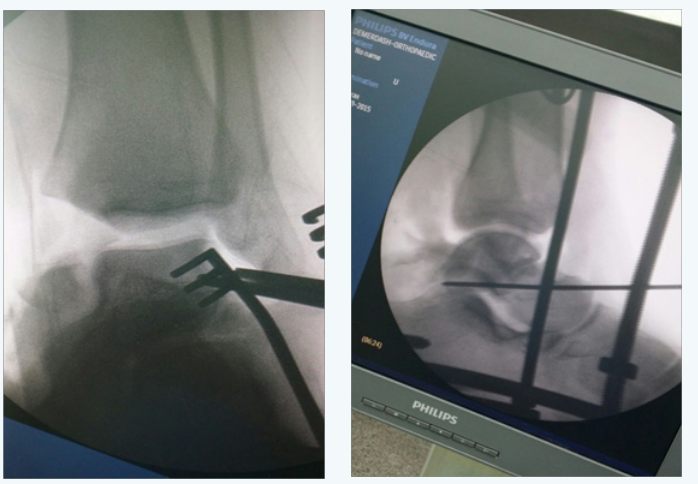

Figure 5: Assessment of reduction.

b. Both rings should be parallel to each other's and to the ankle joint line.

c. Hindfoot alignment should be neutral or seven degrees valgus heel.

Second and third methods of assessment are only applicable if there is no preoperative hindfoot malalignment. Fixation of talar neck is done using two cannulated $4 \mathrm{~mm}$ cancellous screws in anteroposterior direction, one screw through the anteromedial incision and a second screw through the anterolateral incision, both screws should not damage the articular cartilage of the talus. Position of screws is checked using image intensifier (Figure 6). In cases with fractured medial malleolus, it is reduced and fixed using two cannulated $4 \mathrm{~mm}$ screws or $\mathrm{K}$ wires and tension band technique. Closure of wounds done after release of tourniquet and proper haemostasis using 2 zero vicryl sutures (Figure 7).
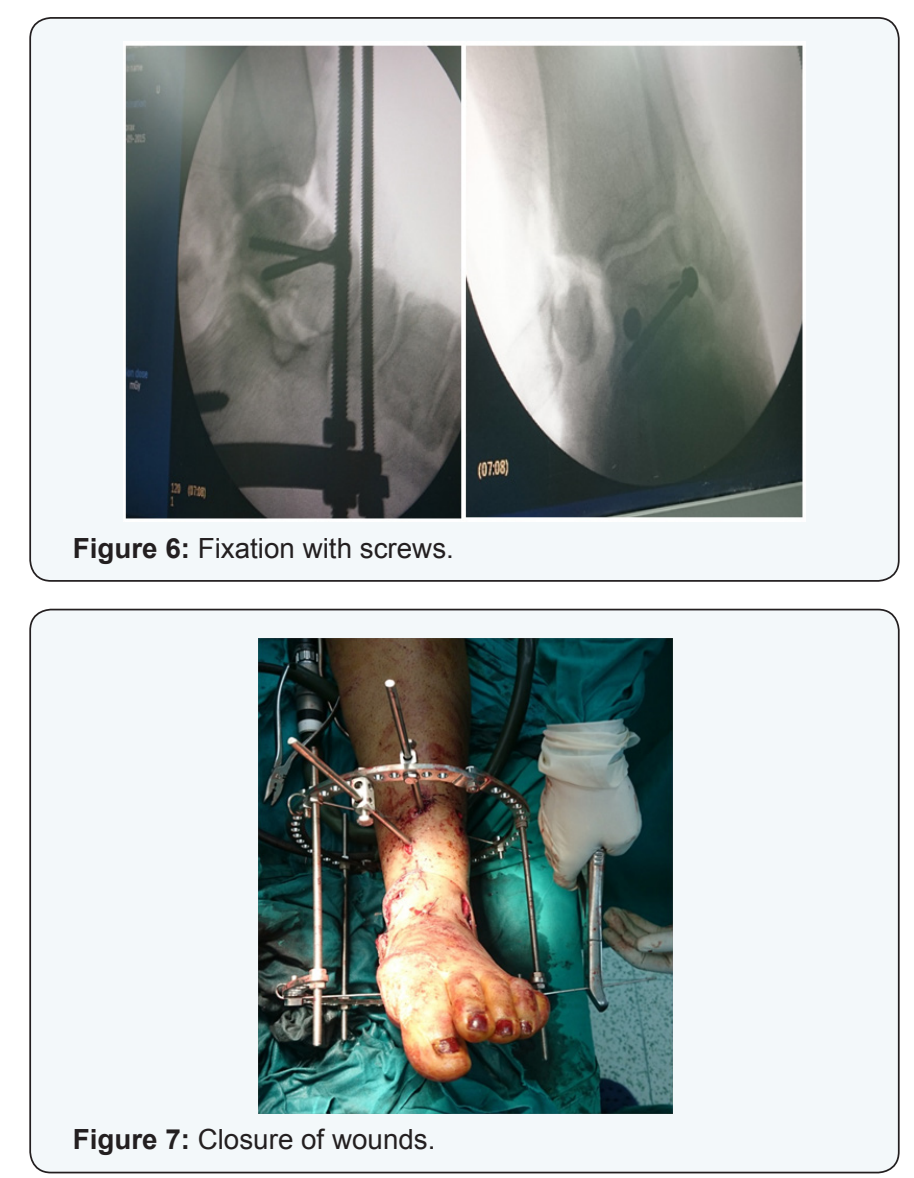

\section{Post operative care}

Plain X ray ankle (anteroposterior, lateral and Mortis view) (Figure 8), CT scan (axial, coronal and sagittal cuts) (Figure 9) were done for all patients to assess accuracy of reduction. Extar care was directed to wounds in order to avoid the possible wound problems especially with the use of double approach (frequent dressing till removal of sutures). Partial weight bearing was allowed at four weeks postoperative, using crutches. Average time for removal of fixator was at 12 weeks .Average follow up time was 12 months. At last follow up visit clinical assessment was done using ankle-hind foot scale of the American Orthopaedic Foot and Ankle Society (AOFAS) to assess functional outcome. The maximum score was 100 (this score was used before by Elgafy et al. [7] to assess patients of talar neck fractures), radiological assessment included plain $\mathrm{x}$ rays. 


\section{Orthopedics and Rheumatology Open Access Journal}

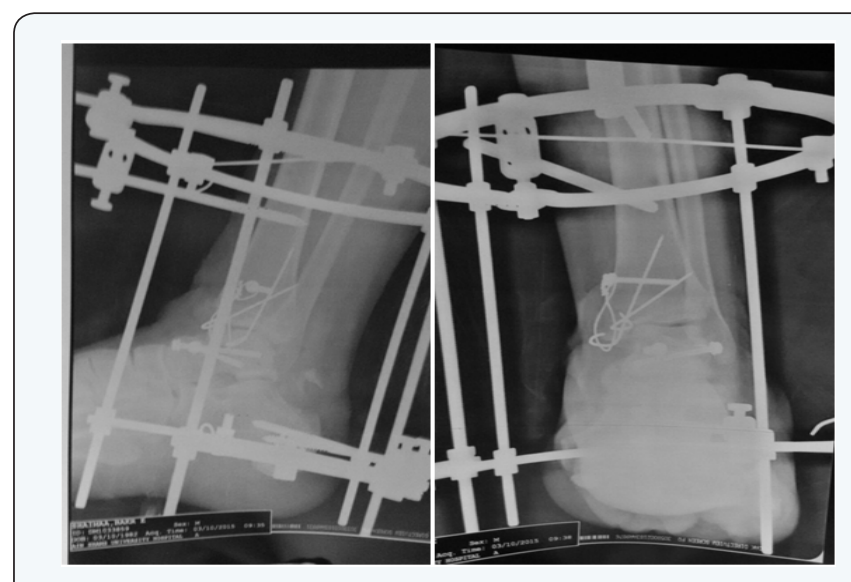

Figure 8: Post operative $X$ rays.

A: Lateral view.

B: Anteroposterior view.

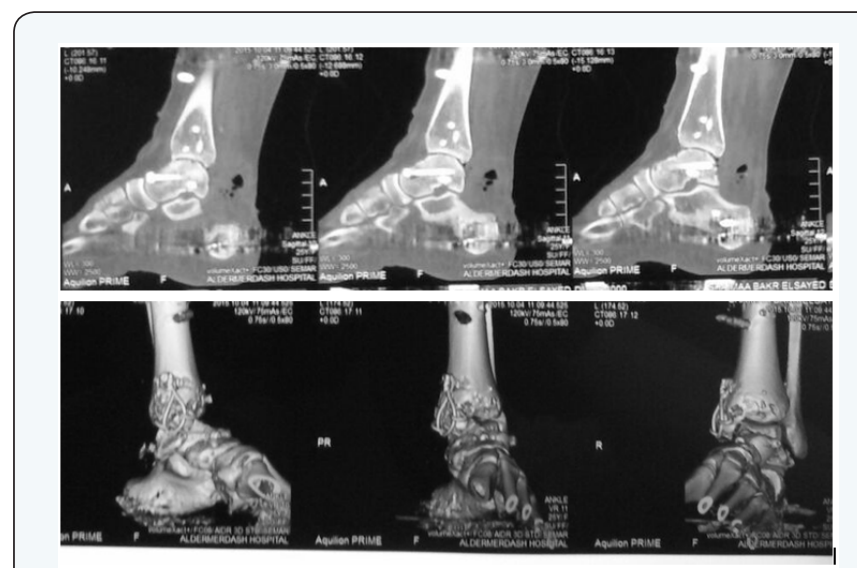

Figure 9: Postoperative CT scan.
A: Sagittal cuts.
B: 3D reconstruction.

\section{Results}

Our series included six patients, four males and two females. Mean age 28 years, Right ankle was affected in four patients and left side in two patients, two cases had open injuries with wound at anteromedial side of ankle, three patients had associated medial malleolar fractures, one patient had posterior tibial artery compromise and its pulse was not felt but immediately after reduction its pulse was palpated well. Average operative time was 85 minutes. All of our patients had achieved good reduction in the postoperative $\mathrm{X}$ rays.

Wound care was satisfactory except for both patients with open wounds at medial side of ankle who had superficial infection and dehiscence that responded well to frequent dressing and parenteral antibiotics.

Fixator was removed at time of clinical union (no tenderness at fracture site and comfortable weight bearing with the fixator), average time for removal of fixator was twelve weeks, in one case fixator removal was delayed due to continuing tenderness at facture site, frame was removed later on (at 16 weeks postoperatively) because of increased pin tract infection, the same case had delayed union and required bone grafting from iliac crest to the medial aspect of the talar neck at six months from operation. Two cases had radiological signs of AVN of talar body (talar body sclerosis) without significant pain and they required no intervention (Table 3 ). No cases required hard ware removal.

All cases at last follow up had decreased range of motion of ankle joint (mean dorsiflexion of five degrees and mean plantar flexion of twenty degrees) this may be attributed not only to the ankle osteoarthritis but also stiffness related to the frame. At last follow up all patients had radiological signs of good union, average functional score was 79.5 out of 100 , three patients had a score of 90 or more with minimal pain, walking unassisted, no effort restrictions, three patients used one crutch occasionally with some discomfort on walking on uneven ground (Table 4) shows details of functional scoring.

Table 4: Functional scoring AOFAS at last follow up.

\begin{tabular}{|c|c|c|c|c|c|c|}
\hline $\begin{array}{c}\text { Patient } \\
\text { no. }\end{array}$ & Pain 40 & $\begin{array}{c}\text { Activity } \\
\text { limitation } \\
10\end{array}$ & $\begin{array}{c}\text { Walking } \\
\text { distance } \\
5\end{array}$ & \multicolumn{2}{|c|}{$\begin{array}{l}\text { Walking } \\
\text { surface } \\
5\end{array}$} & $\begin{array}{c}\text { Ankle } \\
\text { movement } \\
8\end{array}$ \\
\hline 1 & 40 & 10 & 5 & \multicolumn{2}{|c|}{3} & 4 \\
\hline 2 & 40 & 10 & 5 & \multicolumn{2}{|c|}{3} & 4 \\
\hline 3 & 40 & 10 & 4 & \multicolumn{2}{|c|}{3} & 4 \\
\hline 4 & 30 & 7 & 4 & \multicolumn{2}{|c|}{3} & 4 \\
\hline 5 & 30 & 7 & 2 & \multicolumn{2}{|c|}{3} & 4 \\
\hline 6 & 20 & 7 & 2 & \multicolumn{2}{|c|}{3} & 4 \\
\hline $\begin{array}{c}\text { Patient } \\
\text { no. }\end{array}$ & $\begin{array}{l}\text { Subtalar } \\
\text { motion } 6\end{array}$ & $\begin{array}{c}\text { Hind foo } \\
\text { stability } \\
10\end{array}$ & \multicolumn{2}{|c|}{$\begin{array}{l}\text { Alignment } \\
10\end{array}$} & Gait 8 & $\begin{array}{c}\text { Total } \\
\text { score } \\
100\end{array}$ \\
\hline 1 & 3 & 8 & \multicolumn{2}{|c|}{10} & 8 & 91 \\
\hline 2 & 3 & 8 & \multicolumn{2}{|c|}{10} & 8 & 91 \\
\hline 3 & 3 & 8 & \multicolumn{2}{|c|}{10} & 8 & 90 \\
\hline 4 & 3 & 8 & \multicolumn{2}{|c|}{10} & 4 & 73 \\
\hline 5 & 3 & 8 & \multicolumn{2}{|c|}{10} & 4 & 71 \\
\hline 6 & 3 & 8 & \multicolumn{2}{|c|}{10} & 4 & 61 \\
\hline
\end{tabular}

\section{Discussion}

Although many of the complications associated fracture neck talus Hawkin's type III are related to the main insult but some of the postoperative complications start inside the operating theater through the surgical technique and the vigorous manipulation exerted during the procedure so recent authors have stressed the importance of anatomic reduction and the likehood of arthrosis with less than perfect reduction [8]. Vigorous manipulation during reduction leads to harming the delicate blood supply predisposing to AVN of the talus, scuffing of the articular cartilage may lead to early ankle or subtalar 


\section{Orthopedics and Rheumatology Open Access Journal}

osteoarthritis [8] while pushing the extruded talar body back to the tight mortis may cause propagation of the undisplaced cracks adding more comminuted fragments, all of these disadvantages can be avoided by using our technique.

The mean operative time of our technique (85 minutes) is relatively longer than the usual time for doing the open reduction and fixation of talar neck fractures; this may be related to the time consumed in applying the frame. Although medial malleolar osteotomy is recommended in Hawkin's type III talar neck fractures [9] but it is not necessary in our technique because of the role of distraction in making better exposure without needing the osteotomy which adds a new morbidity for the patient. Unlike the other techniques, in our series the reduction could be checked and readjusted freely depending on the frame before fixation with screws, in one case after doing the reduction there was a gap between the talar body and the head in the lateral view, compression could be achieved through translating the foot piece posteriorly in relation to the proximal ring and then fixation was done.

Another tip is to start the fixation with the lateral screw through the anterolateral incison before the medial screw to avoid the overcompression at the medial side leading to varus malalignment of the foot which is common in Hawkin's type III talar neck fractures [6] but it did not happen in any of our cases. Partial weight bearing was allowed for our cases at four weeks after operation which is much earlier than recommended in Hawkin's type III fracture talar neck (recommended 12 to 16 weeks of immobilization and non weight bearing after the operation) [10], this is related to the presence of the frame which acts as a protecting tool against the axial overloading force on the articular cartilage, maintaining the reduction of talar neck and keeping the hind foot alignment, although it may have a role in decreasing the ankle range of motion later on thus we suggest using a hinged fixator in the future to start earlier range of motion even before removal of the frame.

Ankle osteoarthritis did not appear radiologically in any of our patients compared to $25 \%$ of cases in Elgafy [7] series , our technique may has no direct role on decreasing post-traumatic ankle or subtalar arthritis, but we claim that good reduction, respecting the articular cartilage during reducing the extruded talar head back to the mortis in addition to some distraction that happened intraoperatively may have some role in decreasing incidence of osteoarthritis, this needs further follow up of larger number of patients. Radiological signs of AVN of the talar body occurred in two of our patients but they required no further intervention till last follow up, incidence of AVN in Hawkin's type III reported in literature is $75 \%$ [11], this does not mean that AVN is less in our series due to few number of patients, but we believe that achieving perfect reduction with less vigorous manipulation in addition to the protective effect of the frame on the talar dome may have a role in keeping the delicate blood supply of the talus, this needs further vascular studies on large number of patients to prove it. Limitations of the study is the few number of patients, but our main aim is to highlight a new proposed technique to facilitate the reduction and fixation of Hawkin's type III talar neck fractures with nearly extruded talar body.

\section{Conclusion}

Distraction assisted reduction of extruded talar body is a good proposed technique for treatment of Hawkin's type III fractures of neck talus without need for vigorous manipulation. The technique carries a lot of intraoperative and postoperative advantages.

\section{Acknowledgement}

I would like to thank Ain Shams university hospitals in which the study was done and especially orthopedic department which supplied all the technical and scientific support for the study.

\section{References}

1. Juliano P, Dabbah M, Harris TG (2004) Talar neck fractures. Foot Ankle Clinics 9: 723-736.

2. Banks AS, Downey MS, Martin DE, Miller SJ (2001) McGlamry's Comprehensive Textbook of Foot and Ankle Surgery, Volume 1 ( $3^{\text {rd }}$ edn), Lippincott Williams and Wilkins, Philadelphia, USA.

3. Sutpal Singh, Chih-Hui Tsai, Albert Kim, Timothy Dailey (2010) Talar Neck Fracture Reduced and Stabilized with an Ilizarov External Fixator : A case report with three year follow up. The Foot and Ankle Online Journal 3(7): 1.

4. Fortin PT, Balazsy JE (2001) Talus fractures: evaluation and treatment. J Am Acad Orthop Surg 9(2): 114-127.

5. Bucholz RW, Heckman JD, Court-Brown C (2006) (Eds.), Rockwood and Green Fractures in Adults, $\left(6^{\text {th }}\right.$ edn), Lippincott Williams and Wilkins, Philadelphia, USA.

6. Benirschke and Kramer (2014) Talus fractures. Techniques in Orthopaedics 29(1).

7. Elgafy H, Ebraheim NA, Tile M, Stephen D, Kase J (2000) Fractures of the Talus. Foot Ankle Int 21(12): 1023-1029.

8. Schatzker J, Tile M (1993) The management of fractures and Dislocations of the talus. In Tscherne $\mathrm{H}$ and Schatzker J (Eds.), Major Fractures of the Pilon, the Talus, and the Calcaneus: Current Concepts of Treatment. Springer-Verlag, Berlin, Germany, pp: 87-104.

9. Adelaar RS (1990) Fractures of the talus. Instr Course Lect 39: 147156.

10. Higgins TF, Baumgaertner MR (1999) Diagnosis and Treatment of Fractures of the Talus: A Comprehensive Review of the Literature. Foot Ankle Int 20(9): 595-605.

11. Baumhauer JF, Alvarez RG (1995) Controversies in treating talus fractures. Orthop Clin North Am 26(2): 335-351. 